\title{
Effect of Menaquinone-7 (vitamin K2) on vascular elasticity in healthy subjects: results from a one-year study
}

\author{
Cees Vermeer ${ }^{1}$ and Hogne Vik ${ }^{2 *}$ \\ ${ }^{1}$ Cardiovascular Research Institute CARIM, Maastricht University, The Netherlands \\ ${ }^{2}$ NattoPharma ASA, Oslo, Norway
}

\begin{abstract}
Background: Matrix Gla-Protein (MGP) is involved in the prevention of arterial calcification. During vitamin K-insufficiency, MGP is produced in its inactive form: dp-ucMGP. Two 3-year intervention studies in the general population have shown that increased vitamin $\mathrm{K}$ intake may decrease arterial stiffening, but the difference with placebo became only significant in the third year of treatment. In the present trial we have investigated whether in a pre-selected group of vitamin K-insufficient subjects (men and women) an effect of vitamin K-supplementation may be demonstrated within one year.
\end{abstract}

Methods: A randomized placebo-controlled clinical trial was performed in 243 subjects (40-70 years old) characterized by circulating dp-ucMGP concentrations above the median of the general population. Arterial stiffness was concluded from the carotid-femoral pulse-wave velocity (cfPWV), and other vascular characteristics were measured by echotracking of the common carotid artery. Treatment was performed with either vitamin K (menaquinone-7, MK-7) or placebo for one year.

Results: In the total study group, MK-7 induced a significant decrease of both dp-ucMGP and cfPWV. After subdividing by gender, it appeared that the effects were only seen in women, in whom we also found beneficial effects in other vascular characteristics as well as in body weight and BMI.

Conclusions: High vitamin $\mathrm{K}$ intake decreased age-related vascular stiffening. The effects were most obvious in women with poor vitamin $\mathrm{K}$ status and were statistically significant after one year of treatment.

\begin{abstract}
Abbreviations: BMI: Body Mass Index; dp-ucMGP: desphosphouncarboxylated Matrix Gla-Protein; cfPWV: carotid-femoral PulseWave Velocity; crPWV: carotid-radial Pulse-Wave Velocity; IMT: Intima-Media Thickness; CC: Compliance Coefficient; DC: Distensibility Coefficient; E: Young's Modulus
\end{abstract}

\section{Introduction}

Vitamin $K$ functions as an essential cofactor in the posttranslational carboxylation of protein-bound glutamate residues into gammacarboxyglutamate (Gla). Well-known examples of Glacontaining proteins are the blood coagulation factors II, VII, IX and X (all synthesized in the liver) and the extrahepatic proteins osteocalcin and Matrix Gla-Protein (MGP), which are synthesized in bone and in the arterial vessel wall, respectively [1]. The Triage theory posits that, when the availability of a micronutrient is inadequate, nature ensures that micronutrient-dependent functions required for shortterm survival are protected at the expense of functions whose lack has only longer-term consequences [2]. This explains why in the general population the coagulation factors are all fully carboxylated, whereas in most subjects the carboxylation of osteocalcin and MGP is far from complete [3]. The implication of this principle is that vitamin $\mathrm{K}$ intake required for optimal bone and vascular health is higher than that required for normal haemostasis.

Indeed, poor vitamin $\mathrm{K}$ status has been associated with increased risk of cardiovascular disease and mortality. Firstly, population-based studies showed that low dietary vitamin K intake (notably menaquinone, vitamin K2) is associated with arterial calcification and decreased life expectancy [4-6]. Secondly, desphospho-uncarboxylated Matrix Gla-
Protein (dp-ucMGP) - a circulating marker for vitamin K insufficiency - was found to be increased in those at elevated cardiovascular risk. In prospective studies it was shown that in about $40 \%$ of the general population vitamin $\mathrm{K}$-insufficiency (as deducted from circulating dpucMGP) is an independent risk factor for unfavourable cardiovascular outcomes and mortality [7-11]. Thirdly, in a recently published 3-year clinical intervention trial among 244 healthy postmenopausal women, a beneficial effect of vitamin K2 (as menaquinone-7, $180 \mu \mathrm{g} /$ day) on the carotid-femoral pulse-wave velocity (cfPWV) and other markers for vascular health was demonstrated [12]. These health benefits became only statistically significant in the third year of treatment, which implies that long clinical trials are required to demonstrate an effect. Remarkably, the health benefits of supplemental vitamin K2 were most obvious in those with poor vitamin $\mathrm{K}$ status, i.e. circulating dp-ucMGP above the median of the study group ( $400 \mathrm{pmol} / \mathrm{L})$, at baseline.

In the present paper we report a second intervention trial using the same dose of MK-7 (180 $\mu \mathrm{g} /$ day $)$ in a population at elevated risk for cardiovascular disease due to vitamin K-insufficiency (i.e.: dp-ucMGP $>400 \mathrm{pmol} / \mathrm{L}$ ). We hypothesized that in this selected group favourable effects on vascular characteristics may become visible after one year of treatment. Furthermore, this is the first placebo-controlled MK-7 intervention trial in which effects on men and women are compared.

${ }^{\star}$ Correspondence to: Vik H, NattoPharma ASA, PO Box 514, 1327 Lysaker, Norway, Tel: 47 97535326; E-mail: hogne.vik@nattopharma.com

Key words: vitamin K, food supplement, cardiovascular, arteries, clinical trial

Received: June 01, 2020; Accepted: June 13, 2020; Published: June 16, 2020 


\section{Subjects and methods}

\section{Study design}

The study was a double-blind placebo-controlled clinical intervention trial among 243 subjects which was performed by the R\&D Group VitaK, Maastricht University. The required sample size was determined on basis of the MK-7 induced changes in the carotidfemoral pulse-wave velocity (cfPWV) in a previous study [12]. Both men and women were eligible for this study. Inclusion criteria were: age $40-70$ years, body mass index $20-35 \mathrm{~kg} / \mathrm{m} 2$, Caucasian race and circulating dp-ucMGP > $400 \mathrm{pmol} / \mathrm{L}$. Exclusion criteria were: cardiovascular disease, a blood coagulation disorder, hyperlipidaemia, a history of metabolic or gastrointestinal disease, the use more than 3 units of alcoholic beverages per day, and the use of oestrogen replacement (women), corticosteroids, anticoagulants or vitamin K-containing dietary supplements. After randomization (stratified for gender), participants received either MK-7 ( $\mathrm{n}=121,180 \mu \mathrm{g} /$ day) or placebo $(n=122)$ once daily. Compliance to the protocol was monitored by counting the remaining tablets after 6 months and at the end of the study. Blood was taken three times by venepuncture to prepare EDTAplasma: at intake (inclusion/exclusion), at baseline and at the end of the study. The primary endpoint for the study was carotid-femoral pulse-wave velocity (cfPWV), secondary endpoints were carotid artery characteristics as determined by ultrasound. This study was conducted according to the guidelines laid down in the Declaration of Helsinki and all procedures involving human subjects were approved by the Medical Ethics Committee of the Maastricht University (Maastricht, The Netherlands). Written informed consent was obtained from all subjects before entering the study.

\section{Study products}

The supplements used in this study were developed and produced by Legosan AB (Kumla, Sweden). Tablets containing 0 and $180 \mu \mathrm{g}$ of MK-7 (MenaQ7, NattoPharma, Lysaker, Norway) and either 344.786 (placebo) or 344.606 (verum) $\mathrm{mg}$ of micro crystalline cellulose, respectively. Both tablets contained $2 \mathrm{mg}$ silicon dioxide and $3 \mathrm{mg}$ magnesium stearate. An investigator not involved in the study coded the bottles for each participant according to the randomisation list. During each visit 4 bottles ( 50 tablets per bottle) were handed out to the study participants, covering a period of 6 months. To verify the stability of the MK-7 containing tablets the vitamin K content in the tablets was determined every month in duplicate.

\section{Biochemical measurements}

Dp-ucMGP was measured in EDTA-plasma by a precommercial ELISA kit at VitaK (Maastricht University, the Netherlands) [13]. Intraand inter-assay variations were 7.6 and $6.8 \%$, respectively, and the lower detection limit was $50 \mathrm{pmol} / \mathrm{L}$. Dp-ucMGP was measured before intake to determine eligibility to the study. Paired samples (at baseline and at the end of the study) of each subject were assessed on the same ELISA-plate to minimise inter-assay variation. The MK-7 content in the study products was determined as described previously [14]. During the course of the study 2 tablets were taken each month from the same container to test the stability of MK-7 in the tablets.

\section{Regional and local arterial stiffness}

Regional carotid-femoral (cfPWV) and carotid-radial (crPWV) pulse wave velocities were assessed non-invasively by using mechanotransducers directly applied on the skin (Complior, Artech Medical, Pantin, France). The internal yearly Quality Control assessments for the PWV measurements showed variation coefficients of less than $10 \%$.

Echotracking was performed to determine the vascular characteristics of the common carotid artery as described previously [12], using a 7.5 MHz linear array transducer connected to an ultrasound scanner (MyLab One, Esaote, Maastricht, The Netherlands). The following variables were measured: arterial diameter and distensibility (change in diameter from diastole to systole), arterial Distensibility Coefficient (DC) and Compliance Coefficient (CC), Intima Media Thickness (IMT), Young's Elasticity modulus $\mathrm{E}$ and Stiffness Index $\beta$. The yearly Quality Control (inter- and intra-operator CVs) assessments for the measurements of the local arterial stiffness showed coefficients of variation of less than $10 \%$.

\section{Statistical analysis}

The normal distribution for all subjects of the continuous data was analysed by histograms. Log-transformation was performed if variables were not normally distributed (dp-ucMGP). The descriptive statistics (mean and standard deviation of the mean) for each variable at baseline are presented for the total group and per intervention group separately (placebo or vitamin K). Pearson's correlation coefficients were used to investigate the relation between dp-ucMGP at baseline and that after 1 year in the placebo group. The Paired Student t-test was used to test within-group treatment-induced changes between baseline and 1 year. Differences between changes in the placebo group and the MK-7 group were tested using a two-sampled, two-tailed t-test (Welch test). For these analyses we used the statistical package RStudio version 1.1463 (RStudio Inc., Boston, MA, USA). The outcomes of the statistical tests were analysed using a two-sided significance level of $5 \%$. A significance level between 5 and $10 \%$ is considered as indicative of a trend.

\section{Results}

\section{Baseline characteristics}

From the 1302 subjects who responded to the recruitment, 537 subjects returned the questionnaire but 294 of them did not meet the inclusion criteria. The resulting study population of 243 subjects (77 men and 166 women) was randomised into a placebo group $(n=122$, 39 men and 83 women) and an MK-7 group ( $n=121,38$ men and 83 women). At the end of the study, 4 subjects ( 2 men and 2 women) in the placebo group and 4 subjects ( 2 men and 2 women) in the MK-7group had withdrawn from the study. Missing values were dealt with according to the intention-to-treat principle. Seven subjects (4 in the placebo group, 3 in the MK-7 group) reported to experience mild complaints, but only in one case this was a reason for withdrawal from the trial. The compliance to the protocol (intake of study product) was $96.3 \%$ in the MK-7 group and $97.5 \%$ in the placebo group.

The baseline characteristics of the total group, and of the placebo and MK-7 groups separately are given in Table 1. The mean values of the variables at baseline in both treatment groups were comparable ( $p>0.05$ ) except for the Young's Elasticity Modulus E ( $p=0.016)$. The parameters diameter, IMT, CC and BMI in men were significantly different from those in women $(\mathrm{p}<0.0005, \mathrm{p}=0.011, \mathrm{p}=0.011$ and $\mathrm{p}=0.014$, respectively). Stiffness Index $\beta$ showed a strong inverse correlation with distension, DC and CC $(\mathrm{r}=-0.718,-0.930$, and -0.801 , respectively; $\mathrm{p}<0.0005)$.

\section{Study product stability}

During the study period, the stability of MK-7 in the tablets was checked in tablets that were kept under controlled conditions (closed 
Table 1. Baseline characteristics of study population; The Stiffness Index $\beta$ is calculated as $\mathrm{D} / \triangle \mathrm{D} \times \ln (\mathrm{SBP} / \mathrm{DBP})$ where SBP and DBP are the systolic and diastolic blood pressure, $\mathrm{D}$ is the diameter of the carotid artery at diastole and $\Delta \mathrm{D}$ the change in diameter of the carotid artery at systole. P refers to the difference between the placebo group and the MK-7 group

\begin{tabular}{|c|c|c|c|}
\hline Variable & $\begin{array}{l}\text { Total group } \\
\text { Mean (SD) }\end{array}$ & $\begin{array}{l}\text { Placebo group } \\
\text { Mean (SD) }\end{array}$ & $\begin{array}{l}\text { MK-7 group } \\
\text { Mean (SD) }\end{array}$ \\
\hline Number & 243 & 122 & 121 \\
\hline Gender (male/female) & $77 / 166$ & $39 / 83$ & $38 / 93$ \\
\hline Age (years) & $61.0(7.2)$ & $60.7(7.2)$ & $61.3(7.3)$ \\
\hline $\begin{array}{l}\text { Current smoking (\%) } \\
\text { Cigarettes/day }\end{array}$ & $\begin{array}{c}7 \\
9(6)\end{array}$ & $\begin{array}{c}7 \\
8(6)\end{array}$ & $\begin{array}{c}7 \\
10(6)\end{array}$ \\
\hline Former smoking (\%) & 59 & 37 & 62 \\
\hline $\begin{array}{l}\text { Alcohol use (\%) } \\
\text { Units/week }\end{array}$ & $\begin{array}{c}84 \\
6.2(5.0)\end{array}$ & $\begin{array}{c}83 \\
6.1(4.9)\end{array}$ & $\begin{array}{c}86 \\
6.3(5.1)\end{array}$ \\
\hline $\begin{array}{l}\text { Women: } \\
\text { Premenopausal (\%) } \\
\text { Perimenopausal (\%) } \\
\text { Postmenopausal (\%) }\end{array}$ & $\begin{array}{l}24 \\
23 \\
53\end{array}$ & $\begin{array}{l}28 \\
25 \\
47\end{array}$ & $\begin{array}{l}20 \\
20 \\
59\end{array}$ \\
\hline Weight (kg) & $75.2(14.6)$ & $73.9(13.8)$ & $76.6(15.2)$ \\
\hline Height $(\mathrm{cm})$ & $170(8)$ & $169(9)$ & $170(8)$ \\
\hline Body mass index $\left(\mathrm{kg} / \mathrm{m}^{2}\right)$ & $26.0(4.0)$ & $25.8(3.9)$ & $26.2(4.0)$ \\
\hline Dp-ucMGP (pmol/L) & $677(269)$ & $701(309)$ & $653(218)$ \\
\hline $\operatorname{cfPWV}(\mathrm{m} / \mathrm{s})$ & $10.6(2.0)$ & $10.7(2.1)$ & $10.4(1.9)$ \\
\hline $\operatorname{crPWV}(\mathrm{m} / \mathrm{s})$ & $11.1(1.3)$ & $11.1(1.5)$ & $11.0(1.3)$ \\
\hline $\begin{array}{l}\text { Carotid artery: } \\
\text { Diameter }(\mu \mathrm{m}) \\
\text { IMT }(\mu \mathrm{m}) \\
\text { Distension }(\mu \mathrm{m}) \\
\text { DC }\left(\mathrm{MPa}^{-1}\right) \\
\text { CC }\left(\mathrm{mm}^{2} / \mathrm{kPa}\right) \\
\text { Stiffness Index } \beta \\
\text { E }(\mathrm{MPa})\end{array}$ & $\begin{array}{c}7306(893) \\
690(137) \\
416(132) \\
22.0(8.7) \\
0.92(0.37) \\
10.6(3.6) \\
0.56(0.24)\end{array}$ & $\begin{array}{c}7253(953) \\
682135) \\
406(119) \\
22.0(8.6) \\
0.93(0.33) \\
10.6(3.6) \\
0.53(0.22)\end{array}$ & $\begin{array}{c}7359(839) \\
698(140) \\
426(144) \\
22.1(8.9) \\
0.94(0.42) \\
10.5(3.6) \\
0.59(0.26)\end{array}$ \\
\hline
\end{tabular}

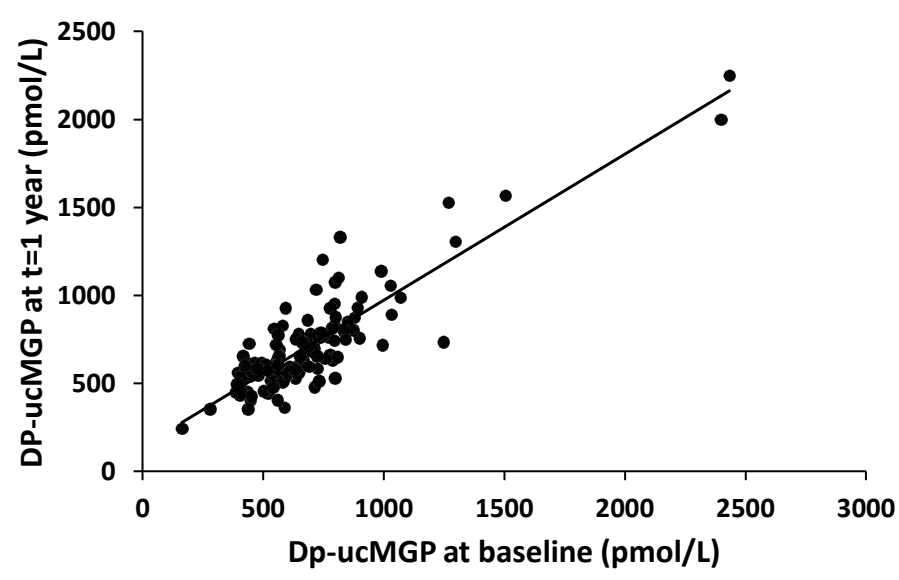

Figure 1. Correlation between circulating dp-ucMGP at baseline and after one year in the placebo group. The linear curve in this figure is defined by the formula [dp-uc $\mathrm{MGP}_{\text {year }}$ ] = $0.83\left[\mathrm{dp}-\mathrm{uc} \mathrm{MGP}_{\text {baseline }}\right]+141$ and $\mathrm{R}^{2}=0.77$

bottles, light-protected and at room temperature). At baseline the mean content was $170 \mu \mathrm{g}$ MK-7/tablet, during the course of the study the MK-7 content steadily decreased to $134 \mu \mathrm{g} /$ tablet. Left-overs returned by the participants were also analysed and occasionally contained even less than $100 \mu \mathrm{g} /$ tablet.

\section{Effects of MK-7 supplementation as measured in the total cohort}

To check the stability of the dp-ucMGP level in time, we have plotted dp-ucMGP at the end of the study as a function of the dpucMGP levels at baseline in the placebo group (Figure 1). It was found that in only two of the 118 participants who completed the study, dpucMGP had decreased substantially (concentrations after 1 year: 278 and $163 \mathrm{pmol} / \mathrm{L}$, respectively) and that in three others dp-ucMGP was between 390 and $400 \mathrm{pmol} / \mathrm{L}$. In all others, the high levels of plasma dp-ucMGP had remained high between intake and baseline, and had also remained high after 1 year of placebo.

The effect of MK-7 treatment is shown in Table 2. As expected, circulating dp-ucMGP decreased, which is indicative for the improved vascular vitamin $\mathrm{K}$ status. The primary endpoint marker cfPWV showed the normal age-related increase in the placebo group, and this increase was statistically significant. In the MK-7 group, however, the difference with baseline was not significant, suggesting a preventive effect of MK-7 administration on this risk factor for cardiovascular disease. Among the other cardiovascular variables measured, Young's elasticity modulus E worsened significantly in the placebo group, and remained constant in the MK-7 group. Diameter, distension, DC, CC and IMT showed more favourable effects in the MK-7 group than in the placebo one, but in these cases the changes did not reach the level of statistical significance.

When analysing the data for men and women separately, it was found that in men the circulating dp-ucMGP concentration had not changed significantly after one year of MK-7 treatment: whereas in the women the mean dp-ucMGP level had decreased from 639 to 450 $\mathrm{pmol} / \mathrm{L}$, in men the decrease was not more than from 681 to $652 \mathrm{pmol} / \mathrm{L}$. Consistently, women also responded more favourably with respect to their vascular characteristics. This is shown in Table 3. Among the women there was a significant decrease of dp-ucMGP in the MK-7 group, versus a non-significant increase in the placebo group. In the placebo group there was a significant increase in cfPWV versus a nonsignificant small increase in the MK-7 group. Most remarkably, IMT decreased significantly in the MK-7 group (and not in the placebo one), whereas distention, DC and CC decreased significantly in the placebo group and not in the MK-7 group. In the male population none of the within-group changes were statistically significant (data not shown).

Table 2. Changes of vascular characteristics in the total study cohort after treatment with either placebo or MK-7

\begin{tabular}{|l|c|c|c|c|}
\hline & \multicolumn{2}{|c|}{ Placebo group } & \multicolumn{2}{c|}{ MK-7 group } \\
\hline Variable & Change (SD) & P & Change (SD) & P \\
\hline Dp-ucMGP $(\mathrm{pmol} / \mathrm{L})$ & $+22(152)$ & 0.11 & $-138(257)$ & $<0.005$ \\
\hline cfPWV $(\mathrm{m} / \mathrm{s})$ & $+0.54(1.76)$ & $<0.005$ & $+0.20(1.86)$ & 0.27 \\
\hline crPWV $(\mathrm{m} / \mathrm{s})$ & $+0.04(1.49)$ & 0.75 & $-0.16(1.18)$ & 0.16 \\
\hline Carotid artery: & & & & \\
Diameter $(\mu \mathrm{m})$ & $+24(601)$ & 0.67 & $-35(250)$ & 0.13 \\
IMT $(\mu \mathrm{m})$ & $+1.07(66.73)$ & 0.86 & $-14.13(85.69)$ & 0.08 \\
Distension $(\mu \mathrm{m})$ & $-21.06(78.11)$ & 0.01 & $-14.22(69.79)$ & 0.03 \\
DC $\left(\mathrm{MPa}^{-1}\right)$ & $-1.59(7.00)$ & 0.02 & $-1.21(5.93)$ & 0.03 \\
CC $\left(\mathrm{mm}^{2} / \mathrm{kPa}\right)$ & $-0.08(0.28)$ & $<0.005$ & $-0.07(0.27)$ & 0.02 \\
Stiffness Index $\beta$ & $+0.37(2.90)$ & 0.16 & $+0.14(2.61)$ & 0.49 \\
E $(\mathrm{MPa})$ & $+0.10(0.32)$ & $<0.005$ & $+0.01(0.33)$ & 0.76 \\
\hline
\end{tabular}

Table 3. Changes of vascular characteristics in women after treatment with either placebo or MK-7

\begin{tabular}{|l|c|c|c|c|}
\hline & \multicolumn{2}{|c|}{ Placebo group } & \multicolumn{2}{c|}{ MK-7 group } \\
\hline Variable & Change (SD) & P & Change (SD) & P \\
\hline Dp-ucMGP $(\mathrm{pmol} / \mathrm{L})$ & $+24(159)$ & 0.18 & $-188(141)$ & $<0.005$ \\
\hline cfPWV $(\mathrm{m} / \mathrm{s})$ & $+0.61(1.66)$ & $<0.005$ & $+0.14(2.10)$ & 0.57 \\
\hline crPWV $(\mathrm{m} / \mathrm{s})$ & $+0.08(1.36)$ & 0.61 & $-0.14(1.20)$ & 0.31 \\
\hline Carotid artery: & & & & \\
Diameter $(\mu \mathrm{m})$ & $-1(250)$ & 0.97 & $-48(250)$ & 0.06 \\
IMT $(\mu \mathrm{m})$ & $+0.80(63.15)$ & 0.91 & $-16.18(73.69)$ & 0.05 \\
Distension $(\mu \mathrm{m})$ & $-19.05(71.86)$ & 0.02 & $-12.16(56.31)$ & 0.06 \\
DC $\left(\mathrm{MPa}^{-1}\right)$ & $-1.58(7.05)$ & 0.05 & $-0.85(6.23)$ & 0.23 \\
CC $\left(\mathrm{mm}^{2} / \mathrm{kPa}\right)$ & $-0.07(0.25)$ & 0.01 & $-0.05(0.27)$ & 0.09 \\
Stiffness Index $\beta$ & $+0.47(2.70)$ & 0.13 & $-0.10(2.35)$ & 0.70 \\
E $(\mathrm{MPa})$ & $+0.06(0.32)$ & 0.32 & $-0.02(0.32)$ & 0.59 \\
\hline
\end{tabular}


We also observed in women (and not in men) that in the MK-7 group there was a weight loss during the year of treatment: the mean body weight decreased from 71.76 to $71.04 \mathrm{~kg}(\Delta=-0.72 \mathrm{~kg}, \mathrm{p}=$ $0.013)$ whereas in the placebo group we observed a minimal and nonsignificant weight decrease from 68.84 to $68.78 \mathrm{~kg}(\Delta=-0.05 \mathrm{~kg} ; \mathrm{p}=$ 0.981. Similarly, the BMI in the MK-7 group declined significantly from 25.77 to 25.48 , whereas in the placebo group there was an insignificant decrease from 25.35 to 25.29 .

\section{Discussion}

In this paper we report that in a selected group of healthy subjects, vitamin K-status (as measured by dp-ucMGP) and cfPWV (the gold standard for arterial elasticity) are both favourably affected by a vitamin K2-containing supplement (MK-7, $180 \mu \mathrm{g} /$ day) during the relatively short treatment period of one year. Other markers of vascular health that were measured all showed a non-significant trend for improvement in the supplemented group as compared to placebo. The effects were mainly seen in women, and are in line with a previous 3-year study among postmenopausal women. It is concluded that effects of vitamin $\mathrm{K}$ administration can be visualized within one year in vitamin K-insufficient subjects at dosages of $180 \mu \mathrm{g} /$ day or higher.

As expected, circulating dp-ucMGP decreased after vitamin $\mathrm{K}$ supplementation. The decrease was less than expected on the basis of a previous dose-response study, in which we found that a dose of $180 \mu \mathrm{g} /$ day of MK-7 induced a decrease by $200 \mathrm{pmol} / \mathrm{L}$ in the normal population [3]. In our present study the decrease at a similar dose was $148 \mathrm{pmol} / \mathrm{L}$. The fact that the effect was slightly lower than expected may be due to the lower stability of the study product (dry tablet) as compared to oilsolubilized MK-7 in previous studies. Also, the fact that the participants in our study were selected to have a poor vitamin $\mathrm{K}$ status may have contributed to the relatively small effect of MK-7 treatment on vascular characteristics, since even at the (intended) daily dose of $180 \mu \mathrm{g} /$ day of MK-7 most subjects remained well above the level of $400 \mathrm{pmol} / \mathrm{L}$ of circulating dp-ucMGP, which was defined as the plasma concentration above which people are at increased risk for cardiovascular disease and mortality [8]. Given the fact that a dose-dependent decrease of dp-ucMGP was found upon MK-7 treatment $[15,16]$, it should be considered, therefore, to increase the dose of supplement to at least 360 $\mu \mathrm{g} /$ day in order to bring the level of circulating dp-ucMGP well below $400 \mathrm{pmol} / \mathrm{L}$ and thus to reach a maximal effect.

('Disclaimer: Vitamin K supplements should not be taken by patients receiving vitamin $\mathrm{K}$ antagonist (oral anticoagulant) treatment.)

\section{Conclusion}

Despite the relatively small improvement of vitamin $\mathrm{K}$ status, a significant benefit of MK-7 was observed on the primary endpoint cfPWV: whereas in the placebo group cfPWV increased in an agedependent way, no increase was observed in the MK-7 treated group. This result is consistent with outcomes in two previous 3-year studies among postmenopausal women [12,17], but to our knowledge this is the first time that a vascular effect was observed within one year of treatment. Remarkably, also the previously reported vitamin K-induced weight loss [18] was only seen in women, The fact that in the previous paper the weight loss was mainly due to the loss of visceral and abdominal fat suggests an additional benefit of high vitamin $\mathrm{K}$ intake.

\section{Acknowledgments}

The authors wish to thank the VitaK staff for expertly executing this trial, and Ms. Hedda Vik for working out all statistics. We also wish to thank NattoPharma ASA for kindly sponsoring this trial.

\section{Conflicts of interest}

Hogne Vik is Chief Medical Officer at NattoPharma. Cees Vermeer (retired since September 2017) has been Chief Scientific Officer at VitaK and Associate Professor of Biochemistry at CARIM, Maastricht University.

\section{References}

1. Willems BAG, Vermeer C, Reutelingsperger CPM, Schurgers LJ (2014) The realm of vitamin K-dependent proteins: shifting from coagulation towards calcification. $\mathrm{Mol}$ Nutr Food Res 58: 1620-1635.

2. McCann JC, Ames BN (2009) Vitamin K, an example of triage theory: is micronutrient inadequacy linked to diseases of aging? Am J Clin Nutr 90: 889-907.

3. Theuwissen E, Magdeleyns EJ, Braam LAJLM, Teunissen KJ, Knapen MHJ, et al (2014) Vitamin K status in healthy volunteers. Food \& Funct 5: 229-234.

4. Geleijnse JM, Vermeer C, Grobbee DE, Pols HAP, Schurgers LJ, et al. (2004) Dietary intake of vitamin K-2 reduces the risk of cardiac events and aortic atherosclerosis: The Rotterdam Study. J Nutr 134: 3100-3105.

5. Gast GCM, de Roos NM, Sluijs I, Bots ML, Beulens JWJ, et al. (2009) A high menaquinone intake reduces the incidence of coronary heart disease. Nutr Metab Cardiovasc Dis 19: 504-510.

6. Beulens JW, Bots ML, Atsma F, Bartelink ML, Prokop M, et al. (2009) High dietary menaquinone intake is associated with reduced coronary calcification. Atherosclerosis 203: 489-493.

7. Van den Heuvel EGHM, van Schoor NM, Lips P, Magdeleyns EJP, Deeg DJH, et al. (2014) Circulating uncarboxylated Matrix Gla protein, a marker of vitamin K status, as a risk factor of cardiovascular disease. Maturitas 77: 137-141.

8. Liu Y-P, Gu Y-M, Thijs L, Knapen MHJ, Salvi E, et al. (2015) Inactive Matrix Gla Protein is causally related to health outcomes: a Mendelian randomization study in a Flemish population. Hypertension 65: 463-470.

9. Pivin E, Ponte B, Pruijm M, Ackermann D, Guessous I, et al. (2015) Inactive matrix GLA protein is associated with arterial stiffness. Hypertension 66: 85-92.

10. Riphagen IJ, Keyzer CA, de Borst M, Gansevoort RT, Geleijnse JM, et al. (2017) Prevalence and effects of functional vitamin $\mathrm{K}$ insufficiency in the general population: The PREVEND study. Nutrients 9: 1334.

11. Wei F-F, Trenson S, Monney P, Yang W-Y, Pruijm M, et al. (2018) Epidemiological and histological research implicate matrix Gla protein in diastolic left ventricular dysfunction. PloS One 13: e0193967.

12. Knapen MHJ, Braam LAJLM, Drummen NEA, Bekers O, Hoeks APG, et al. (2015) Low-dose menaquinone-7 supplementation improves vascular properties in healthy postmenopausal women. Thromb Haemostas 113: 1135-1144.

13. Cranenburg ECM, Koos R, Schurgers LJ, Magdeleyns E, Schoonbrood THM, et al. (2010) Characterisation and potential diagnostic value of circulating matrix Gla protein (MGP) species. Thromb Haemostas 104: 811-822.

14. Schurgers LJ, Vemeer C (2000) Determination of Phylloquinone and Menaquinones in Food: Effect of Food Matrix on Circulating Vitamin K Concentrations. Haemostasis 30: 298-307.

15. Dalmeijer GW, van der Schouw YT, Magdeleyns E, Ahmed N, Vermeer C, et al. (2012) The effect of menaquinone -7 supplementation on circulating species of matrix-Gla protein. Atherosclerosis 225: 397-402.

16. Caluwé R, Vandecasteele S, Van Vlem B, Vermeer C, De Vriese AS (2014) Vitamin K2 supplementation in hemodialysis patients: a randomized dose-finding study. Nephrol Dial Transpl 29: 1385-1390.

17. Braam LAJLM, Hoeks APG, Brouns F, Hamulyák K, Gerichhausen MJW, et al (2004) Beneficial effects of vitamin $\mathrm{K}$ on the elastic properties of the vessel wall in postmenopausal women: a follow-up study. Thromb Haemostas 91: 373-380.

18. Knapen MHJ, Drummen NEA, Jordan K, Vermeer C (2018) Vitamin K-induced effects on body fat and weight: results from a 3-year vitamin $\mathrm{K} 2$ intervention study. Eur J Clin Nutr 72: 136-141.

Copyright: (C2020 Vermeer C. This is an open-access article distributed under the terms of the Creative Commons Attribution License, which permits unrestricted use, distribution, and reproduction in any medium, provided the original author and source are credited. 